planetary and Magnetospheric Observations, Saulgau, W. Germany, 22-25 May 1973 (to be published).

${ }^{10}$ F. A. Hanser, B. Sellers, and P. R. Morel, U. S. Air Force Cambridge Research Laboratories Report
No. AFCRL-TR-73-0144, 1973 (unpublished).

${ }^{11}$ A. B. Severny, Izv. Krym. Astrofiz. Observ. 26 , 12 (1956).

${ }^{12}$ J. D. Anglin, Bull. Amer. Phys. Soc. 18, 696 (1973).

\title{
Mass Limit on a Positively Charged Heavy Muon*
}

B. C. Barish, J. F. Bartlett, D. Buchholz, T. Humphrey, F. S. Merritt, X. Nagashima, $\nmid$ F. J. Sciulli, D. Shields, and H. Suter $末$

California Institute of Technology, Pasadena, California 91109

and

G. Krafczyk and A. Maschke§

National Accelerator Laboratory, Batavia, Ilinois 60510

(Received 6 June 1973)

\begin{abstract}
We report a preliminary search for a positively charged lepton $\left(Y^{+}\right)$coupled directly to the $\mu^{-}$and $\nu_{\mu}\left(i_{\circ} e_{\circ}\right.$, muon number $\left.=+1\right)$. Using the production process $\nu_{\mu}+N \rightarrow Y^{+}$ +anything, we have looked for the $\mu^{+}$from the decay $Y^{+} \rightarrow \mu^{+}+\nu_{\mu}+\nu_{\mu}$. The expected number of $\mu^{+}$events is given as a function of the heavy-lepton mass, the branching fraction to muons, and the heavy-lepton coupling. For typical gauge-theory predictions, we obtain the $90 \%$ confidence limit $M_{\boldsymbol{Y}}>2 \mathrm{GeV} / c^{2}$.
\end{abstract}

One striking feature of weak interactions is the small number of members observed in the known lepton families. For example, the muon family presently contains only two observed members: $\nu_{\mu}$ and $\mu^{-}$. It is important to search for higher mass members, whose existence could have profound implications.

Considerable theoretical work ${ }^{1,2}$ in the past few years has focused on unified gauge theories to predict controlled high-energy behavior for the weak interactions and to unify the theoretical treatment of weak and electromagnetic interactions. Theories suggested thus far contain massive charged intermediate vector bosons $\left(M_{w}\right.$ $\left.\sim 40 \mathrm{GeV} / c^{2}\right)$ together with neutral weak currents and/or heavy leptons. The suggested heavy leptons have the following properties: (1) They belong to existing lepton families (either electron or muon), and (2) they have different electric charge from existing charged leptons of the same lepton number.

The phenomenology of the proposed particles has been discussed at length by Bjorken and Llewllyn-Smith (BLS) ${ }^{3}$ and by Llewellyn-Smith. ${ }^{4}$ These authors mention several ways of searching for massive leptons. To date, we know that any heavy lepton must have a mass greater that that of the $K$ meson. Some calculation $\mathrm{s}^{3,5}$ have indicated that, were a positive heavy muon to exist with mass less than $1 \mathrm{GeV} / c^{2}$, it might have been observed in the CERN neutrino data. ${ }^{6}$

We emphasize here the production of heavy muons $\left(Y^{+}\right)$, in a beam of high-energy muon neutrinos. The ordinary mode of interaction for neutrinos of high energy is

$$
\nu_{\mu}+N \rightarrow \mu^{-}+\text {hadrons. }
$$

The conjectured $\mathrm{Y}^{+}$would be produced by the process

$$
\nu_{\mu}+N \rightarrow Y^{+}+\text {hadrons. }
$$

Reaction (1) has been observed and measured at $\mathrm{CERN}^{6}$ with neutrinos of energy $E_{\nu} \approx 1-10 \mathrm{GeV}$. The results are consistent with neutrino scattering from spin- $\frac{1}{2}$ fractionally charged pointlike constituents (quarks), with predominantly $V-A$ coupling (mainly scattering off quarks rather than antiquarks). ${ }^{7}$ Preliminary National Accelerator Laboratory data are also qualitatively consistent with this picture at higher energies. ${ }^{8.9}$

In the quark model, the differential cross section for either (1) or (2) is given by

$$
\frac{d^{2} \sigma_{ \pm}}{d x d y}=\frac{g_{ \pm}^{2} s}{\pi\left(s x y+M_{w}{ }^{2}\right)^{2}}\left[2 x f(x)\left(1-\frac{m_{ \pm}^{2}}{s x}\right)+2 x \bar{f}(x)(1-y)\left(1-y-\frac{m_{ \pm}^{2}}{s x}\right)\right],
$$




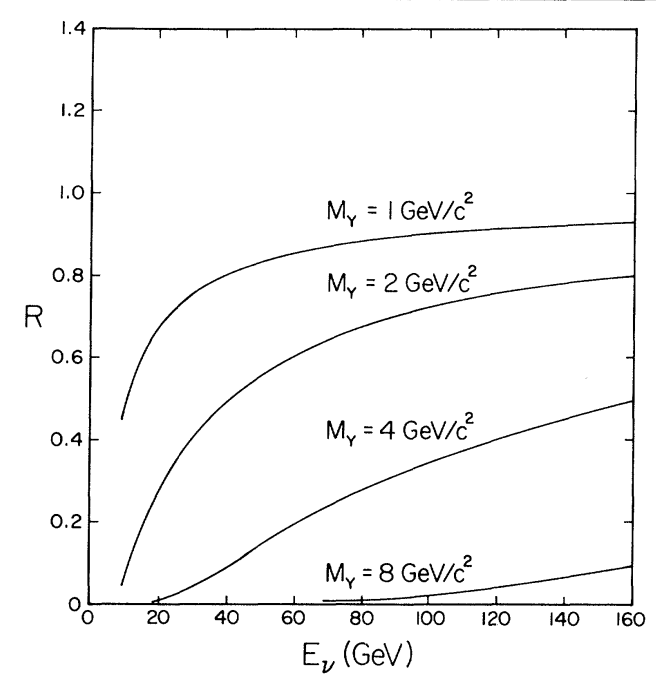

FIG. 1. Ratio of $Y^{+}$production to $\mu^{-}$production in a beam of muon neutrinos of energy $F_{\nu}$. The quark-model structure function relationships are assumed.

where $g_{+}\left(g_{-}\right)$is the coupling of the $Y^{+}\left(\mu^{-}\right)$to the muon neutrino; $s$ is the square of the center-ofmass energy; $M_{w}$ is the mass of the charged intermediate vector boson (assumed large); $x, y$ are the deep-inelastic variables $\left(x=Q^{2} / 2 M \nu, y\right.$ $\left.=\nu / E_{\nu}\right) ; 2 x f(2 x \bar{f})$ is the momentum distribution for quarks (antiquarks) in the nucleon (related to the deep-inelastic structure functions); and $m_{+}\left(m_{-}\right)$is the mass of the $Y^{+}\left(\mu^{-}\right)$. Figure 1 shows the ratio of total cross sections expected for Reactions (2) and (1), obtained by integrating Eq. (3) over the available phase space, and assuming that $g_{+}{ }^{2}=g_{-}{ }^{2}$. For this calculation, we have taken $x f(x) \propto F_{2}(x)^{e d}$, i.e., the scaling structure function found in inelastic electron-deuteron scattering, ${ }^{10}$ and $\bar{f}(x)=0$. Inclusion of the small antiquark component ${ }^{7}$ implied by the low-energy CERN neutrino data changes Fig. 1 by a negligible amount. The calculation also assumes that $M_{w}{ }^{2} \gg s$.

The decay modes expected for the $Y^{+}$are

$$
\begin{aligned}
& Y^{+} \rightarrow \nu_{\mu}+\mu^{+}+\nu_{\mu}, \\
& Y^{+} \rightarrow \nu_{\mu}+e^{+}+\nu_{e}, \\
& Y^{+} \rightarrow \nu_{\mu}+\text { hadrons. }
\end{aligned}
$$

The search described here is for $\mu^{+}$from the decay (4). If these are the only modes available, it has been estimated that ${ }^{3}$

$$
B \equiv \Gamma\left(Y^{+} \rightarrow \nu_{\mu}+\mu^{+}+\nu_{\mu}\right) / \Gamma\left(Y^{+} \rightarrow \text { anything }\right) \simeq 0.3
$$

for $1<M_{y}<6 \mathrm{GeV} / c^{2}$. However, if there exists an uncharged heavy muon, $Y^{0}$, which is lighter than the $Y^{+}$, other decays of the type $Y^{+} \rightarrow Y^{0}$ +anything might be expected. The exact contribution is somewhat model- and mass-dependent, though phase-space considerations alone should make such decay rates small in comparison to (4), (5), and (6).

In our experiment, a narrow-band, sign-selected beam of positive pions and kaons, of mean energy $160 \mathrm{GeV}$, was allowed to decay to neutrinos $\left(\pi^{+} \rightarrow \mu^{+}+\nu_{\mu}\right.$ or $\left.K^{+} \rightarrow \mu^{+}+\nu_{\mu}\right)$. The forward neutrinos entered our apparatus, giving two bands of neutrino energy: from $K$ decay, $\left\langle E_{\nu}\right\rangle \simeq 145$ $\mathrm{GeV}$; from $\pi$ decay, $\left\langle E_{\nu}\right\rangle \simeq 50 \mathrm{GeV}$. This run has been described previously ${ }^{11}$ in a communication describing a search for the charged intermediate boson. The same data, consisting of 112 reconstructed neutrino interactions, are used in the present paper. For this search, the signselected nature of the beam is of prime importance. The only reaction that might be expected to produce a background $\mu^{+}$in our sample is

$$
\bar{\nu}_{\mu}+N \rightarrow \mu^{+}+\text {hadrons }
$$

from background $\pi^{-}$or $K^{-}$decays.

The run reported here consisted of $2.65 \times 10^{16}$ protons on target. Of the 112 events inside the fiducial volume which could be reconstructed kinematically, 111 had a definite $\mu^{-}$secondary. Subsequent analysis ${ }^{8}$ showed that these events are consistent with production from Reaction (1), assuming quark-model structure functions.

There existed in this run a single $\mu^{+}$event with observed hadron energy $E_{h} \simeq 2 \mathrm{GeV}$, muon energy $E^{\prime}=57 \mathrm{GeV}$, and muon angle $\theta^{\prime}=34 \mathrm{mrad}$. If this event corresponded to decay of a $Y^{+}$, its mass would be several $\mathrm{GeV} / c^{2}$. However, we believe that this event comes from $\bar{\nu}_{\mu}$ contamination (7) for the following reasons:

(1) Independent background runs demonstrated the existence of wide-band antineutrino background at roughly the one-event level.

(2) This event has small hadron energy. This is more characteristic of $\bar{\nu}$ interactions than of heavy-lepton production. Figure 2 shows the fraction of $\mu^{+}$events expected, for both cases, in which the ratio $(y)$ of observed hadron energy to observed total energy is less than $y_{u}$. The curves include the apparatus acceptance, and incorporate the cross section (3). The observed event had $y=0.03$. About $1 \%$ of $Y^{+}$decays might be expected to have $y \leqslant 0.03$, while about $20 \%$ of $\bar{\nu}$ interactions are expected with $y \leqslant 0.03$. This occurs principally because the secondary $\mu^{+}$in $\bar{\nu}_{\mu}$ interactions takes a large fraction of the en- 


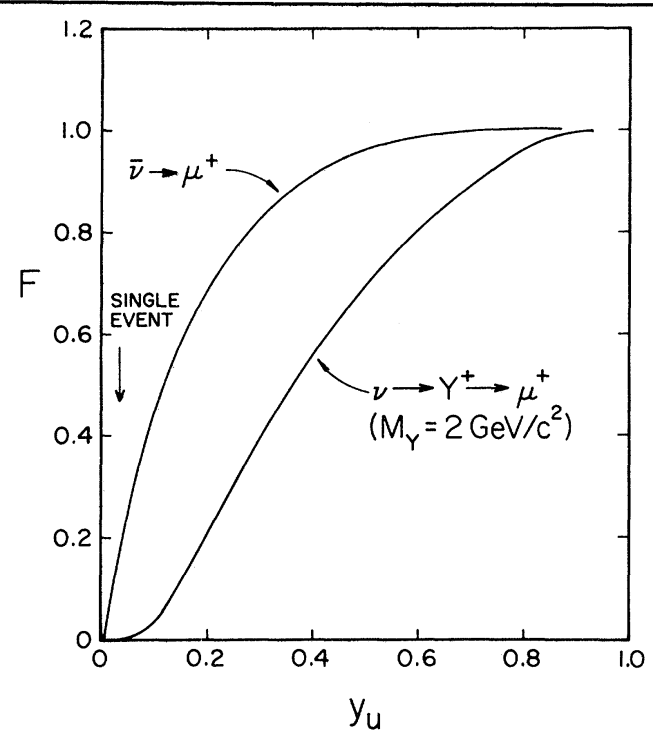

FIG. 2. The calculated fraction, $F$, of events in our apparatus with $y<y_{u}$, where $y=E_{h} / E_{\text {tot }}$. The curves correspond to the reactions

$$
\begin{aligned}
& \nu+N \rightarrow Y^{+}+\text {anything, } \\
& \mathrm{L}_{\mu^{+}}+\nu_{\mu}+\nu_{\mu}
\end{aligned}
$$

and

$$
\bar{\nu}+N \rightarrow \mu^{+} \text {anything. }
$$

Quark-model structure functions are assumed. The single observed $\mu^{+}$event is indicated.

ergy, ${ }^{7}$ while the $\mu^{+}$from the predominantly lefthanded $Y^{+}$has low laboratory energy. We conclude that this anomalous event is most likely from $\bar{\nu}_{\mu}$ contamination.

To calculate our sensitivity, we have used a Monte Carlo calculation which assumes the cross section (3), and takes into account the polarization of the $Y^{+}$and subsequent decay angular distribution via Reaction (4). Figure 3 shows $N\left(g_{-} /\right.$ $\left.g_{+}\right)^{2}(0.3 / B)$, where $N$ is the number of $\mu^{+}$events expected in this run, as a function of heavy lepton mass, $M_{\mathrm{Y}}$. For simple gauge theories, we have $g_{+}{ }^{2}=g_{-}{ }^{2}$, and for specific assumptions for the decay probability, ${ }^{3}$ we have $B \simeq 0.3$. With these assumptions, the ordinate corresponds to the expected number of events in the experiment. The one-event level is in the region of $M_{Y} \sim 5$ $\mathrm{GeV} / c^{2}$. The Poisson probability for observing one event when the mean is 3.56 is equal to 0.1 . We can therefore quote a lower mass bound on a $Y^{+}$as $M_{Y}>2 \mathrm{GeV} / c^{2}$, with $90 \%$ confidence. For differing assumptions regarding the lepton coupling or branching ratio, the limit can be adjusted.

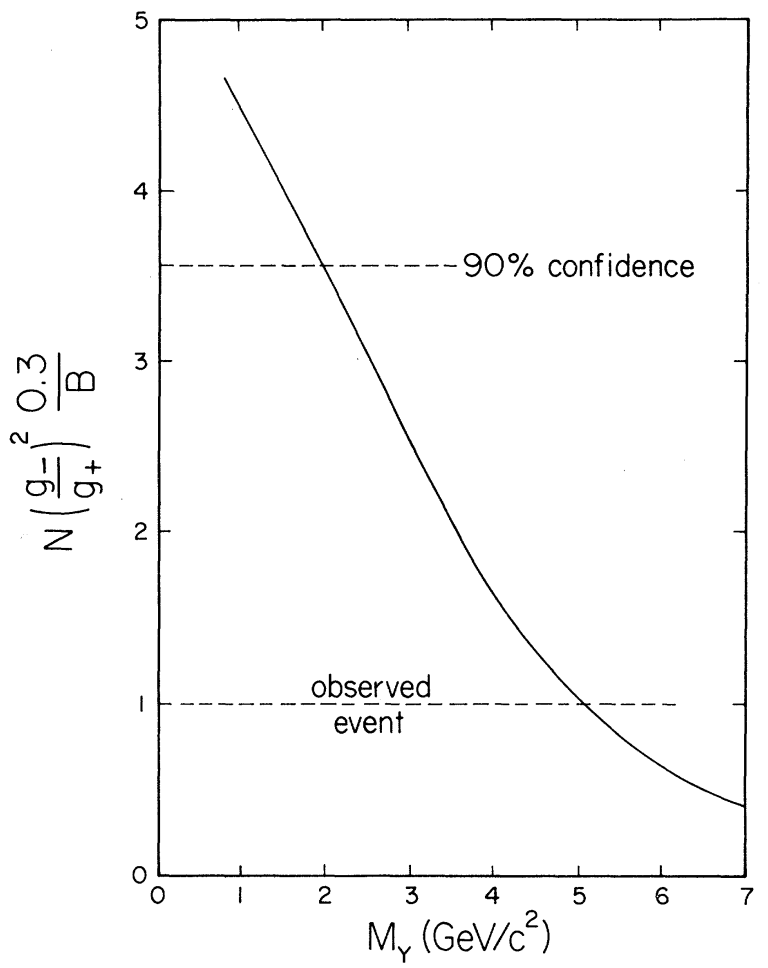

FIG. 3. $N\left(g_{-} / g_{+}\right)^{2} 0.3 / B$ as a function of heavy lepton mass, $M_{Y} \circ N$ is the expected number of $\mu^{+}$events in the experiment, $B$ is the branching ratio for $Y^{+} \rightarrow \nu_{\mu}$ $+\mu^{+}+\nu_{\mu}$, and $g_{+}\left(g_{-}\right)$is the weak coupling of the heavy lepton (muon) to the neutrino and the weak current.

We appreciate the support of the National Accelerator Laboratory staff, in particular members of the Neutrino Laboratory.

*Work supported in part by the U. S. Atomic Energy Commission. Prepared under Contract No. AT (11-1)68 for the San Francisco Operations Office, U. S. Atomic Energy Commission.

$\dagger$ Present address: National Laboratory for High Energy Physics, Oho-Machi, Tsukuba-gun, Ibaraki-ken, 300-32, Japan.

\$Swiss National Fund for Scientific Research Fellow. $\$$ Present address: Brookhaven National Laboratory, Upton, N. Y. 11792.

${ }^{1}$ S. Weinberg, Phys. Rev. Lett. 19, 1264 (1967); A. Salam, in Elementary Particle Theory, edited by N. Svartholm (Almquist and Forlag A. B., Stockholm, 1968).

${ }^{2}$ For a review of gauge theories and references, see B. W. Lee, in Proceedings of the Sixteenth International Conference on High Energy Physics, The University of Chicago and National Accelerator Laboratory, 1972, edited by J. D. Jackson and A. Roberts (National Accelerator Laboratory, Batavia, Ill., 1973), Vol. 4, p. 249.

${ }^{3}$ J. D. Bjorken and C. H. Llewellyn-Smith, SLAC Re- 
port No. SLAC-PUB-1107, 1972 (unpublished).

${ }^{4}$ C. H. Llewellyn-Smith, CERN Report No. CERN-Th1621, 1973 (unpublished).

${ }^{5}$ S. S. Gerstein and V. N. Folomeshkin, Yad. Fiz. 8 , 768 (1969) [Sov. J. Nucl. Phys. $\underline{8}, 447$ (1969)].

${ }^{6} \mathrm{Ph}$. Heusse et al., in Proceedings of the Sixteenth International Conference on High Energy Physics, The University of Chicago and National Accelerator Laboratory, 1972, edited by J. D. Jackson and A. Roberts (National Accelerator Laboratory, Batavia, 111., 1973), paper No. 783 .

${ }^{7}$ D. H. Perkins, in Proceedings of the Sixteenth International Conference on High Energy Physics, The University of Chicago and National Accelerator Laboratory, 1972, edited by J. D. Jackson and A. Roberts (National Accelerator Laboratory, Batavia, Ill., 1973), Vol. 4, p. 211.

${ }^{8}$ B. C. Barish, J. F. Bartlett, D. Buchholz, T. Humphrey, F. Merritt, Y. Nagashima, F. J. Sciulli, D. Shields, H. Suter, G. Krafczyk, and A. Maschke, Caltech Report No. CALT-68-396, 1973 (to be published).

${ }^{9}$ A. Benvenuti, D. Chang, D. Cline, W. T. Ford, R. Imlay, T. Y. Ling, A. K. Mann, F. Messing, J. Pilcher, D. D. Reeder, C. Rubbia, and L. Sulak, Phys. Rev. Lett. 30, 1084 (1973).

${ }^{10} \mathrm{~A}$. Bodek, thesis, Massachusetts Institute of Technology Report No. COO-3069-116, 1973 (unpublished).

${ }^{11}$ B. C. Barish, J. F。 Bartlett, D. Buchholz, T. Humphrey, F. Merritt, Y. Nagashima, F. J. Sciulli, D. Shields, H. Suter, G. Krafczyk, and A. Maschke, Phys. Rev. Lett. 31, 180 (1973).

\title{
Inclusive $\pi^{-}$Distributions from $205-\mathrm{GeV} / c p p$ Interactions*
}

\author{
Y. Cho, R. Engelmann, $\dagger$ T. Fields, L. Hyman, and R. Singer \\ Argonne National Laboratory, Argonne, Illinois 60439
}

and

L. Voyvodic and J. Whitmore

National Accelerator Laboratory, Batavia, Ilinois 60510

(Received 11 June 1973)

\begin{abstract}
Inclusive $\pi^{-}$distributions from $205-\mathrm{GeV} / c$ pp interactions, covering the full range of longitudinal and transverse momenta, have been obtained using the 30-in. hydrogen bubble chamber at the National Accelerator Laboratory. These data provide new evidence for scaling in the fragmentation region and for a plateau in the central region of rapidity. The $90^{\circ}$-c.m. invariant cross section varies as $\exp \left[-(10 \pm 2) P_{T}{ }^{2}\right]$ in the range $P_{T}{ }^{2} \leqslant 0.1$ $(\mathrm{GeV} / c)^{2}$.
\end{abstract}

In this Letter, we report on a measurement of the single-particle inclusive distribution for the reaction $p p \rightarrow \pi^{-}+$anything at $205 \mathrm{GeV} / c$. This measurement covers the entire range in both the center-of-mass longitudinal $\left(P_{L}{ }^{*}\right)$ and transverse $\left(P_{T}\right)$ momenta. This is also the highest energy at which a single experiment covering the entire range of these variables has been reported. Similar data $^{1,2}$ have been reported from the CERN intersecting storage rings (ISR). These ISR experiments cover various restricted intervals of $P_{L}$ * and $P_{T}$, at energies corresponding to laboratory momenta between 225 and $1500 \mathrm{GeV} / c$.

Our data were obtained from approximately 17000 pictures taken with the 30-in. hydrogen bubble chamber exposed to a $205-\mathrm{GeV} / c$ proton beam at the National Accelerator Laboratory (NAL). The results reported here are confined to negative-particle production. Ordinarily, the measurement of momenta and angles of secondary tracks in a bubble chamber is a routine matter. However, for this high beam momentum and for high-multiplicity events, there are practical complications involving track matching in the different stereoscopic views, overlapping track images, and poor momentum determination for fast forward-going secondaries. To determine how the se complications might affect our data, we have made two independent sets of measurements, using somewhat different experimental methods.

Following the $\operatorname{scan}^{3}$ of the film, a fiducial volume was selected that yielded a $20-\mathrm{cm}$ minimum track length for outgoing tracks. For the first measurement method, each view was examined at high magnification (6 times life size), and the spatial pattern of the bubbles was used to match all secondary tracks. The event was then measured in two views and spatially reconstructed using TVGP. For the second method, a minimumsagitta criterion was used to determine which 